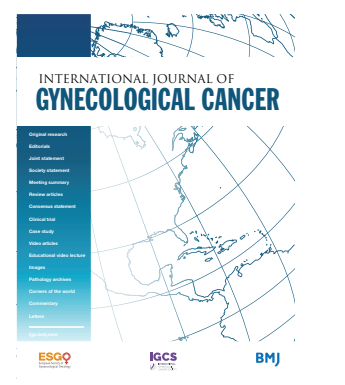

\title{
Role of adjuvant radiation therapy after radical hysterectomy in patients with stage IB cervical carcinoma and intermediate risk factors
}

Dimitrios Nasioudis (D) , Nawar A Latif, Robert L Giuntoli II, Ashley F Haggerty, Lori Cory, Sarah H Kim, Mark A Morgan, Emily M Ko

Division of Gynecologic Oncology, Penn Medicine, Philadelphia, Pennsylvania, USA

Correspondence to Dr Dimitrios Nasioudis, Obstetrics and Gynecology, Hospital of the University of Pennsylvania, Philadelphia, PA 19104, USA; dimitrios. nasioudis@uphs.upenn.edu

Received 1 February 2021 Revised 15 April 2021 Accepted 16 April 2021 Published Online First 6 May 2021
Check for updates

(C) IGCS and ESG0 2021. No commercial re-use. See rights and permissions. Published by BMJ.

\begin{tabular}{l}
\hline To cite: Nasioudis D, Latif NA, \\
Giuntoli II RL, et al. Int J \\
Gynecol Cancer 2021;31:829- \\
834.
\end{tabular}

\section{HIGHLIGHTS}

- 765 cases of stage IB cervical carcinoma with intermediate risk features identified.

- Approximately $50 \%$ received adjuvant beam radiotherapy.

- There was no difference in overall survival between observation and adjuvant beam radiotherapy.

\section{INTRODUCTION}

Objective To investigate the outcomes of observationalone versus adjuvant radiotherapy for patients with lymph node negative FIGO 2018 stage IB cervical carcinoma following radical hysterectomy with negative prognostic factors.

Methods The National Cancer Database was accessed and patients with no history of another tumor, diagnosed with intermediate risk (defined as tumor size $2-4 \mathrm{~cm}$ with lymph-vascular invasion or tumor size $>4 \mathrm{~cm}$ ) pathological stage IB squamous, adenosquamous carcinoma or adenocarcinoma of the cervix between January 2010 and December 2015 who underwent radical hysterectomy with lymphadenectomy and had negative tumor margins were identified. Overall survival was assessed following generation of Kaplan-Meier curves and compared with the log-rank test. A Cox model was constructed to control for a priori selected confounders known to be associated with overall survival.

Results A total of 765 patients were identified and adjuvant external beam radiotherapy was administered to 378 patients $(49.4 \%)$. There was no difference in overall survival between patients who did and did not receive adjuvant radiotherapy, $\mathrm{P}=0.44$ : 4 -year overall survival rates were $88.4 \%$ and $87.1 \%$ respectively. After controlling for patient age, histology, and surgical approach, the administration of adjuvant radiotherapy was not associated with better survival (HR $0.86,95 \% \mathrm{Cl} 0.54$ to 1.38 ). For patients who received adjuvant radiotherapy, there was no survival difference between those who did $(n=219)$ and did not $(n=159)$ receive concurrent chemotherapy, $P=0.36$ : 4-year overall survival rates were $89.8 \%$ and $86.3 \%$, respectively.

Conclusion In a large cohort of patients with lymph node negative, margin negative, stage IB cervical carcinoma, with negative prognostic factors, the administration of adjuvant external beam radiation therapy was not associated with a survival benefit compared with observation alone.
Cervical cancer is the third most common gynecologic malignancy in the United States with a decreasing annual incidence. ${ }^{1}$ However, approximately 13800 women were diagnosed with cervical cancer in 2020. ${ }^{12}$ For patients presenting with early-stage disease, primary radical hysterectomy with lymphadenectomy is preferred while those with locally-advanced disease are administered definitive chemoradiation. $^{34}$

Following radical surgery, patients deemed intermediate risk based on a combination of risk factors that includes presence of lymph-vascular invasion, large tumor size, and depth of stromal invasion, are offered adjuvant radiation therapy to reduce the risk of local relapse. ${ }^{34}$ This recommendation is based on data derived from the Gynecologic Oncology Group 92 landmark randomized trial, that demonstrated improvement in progression-free survival with receipt of adjuvant radiation over observation following radical hysterectomy and lymphadenectomy for margin negative, lymph node negative early-stage cervical cancer. ${ }^{5}$ However, radiation therapy is associated with significant morbidity and can have a significant impact on the quality of life of patients. ${ }^{6}$

Recent retrospective studies suggest that observation alone may potentially be an acceptable option with excellent local control. ${ }^{78}$ The aim of this study was to investigate the outcomes of observation-alone vs adjuvant radiation with or without chemotherapy for patients with lymph node negative intermediate risk FIGO 2018 stage IB cervical carcinoma following radical hysterectomy using a large hospital-based database. 


\section{Original research}

\section{METHODS}

The National Cancer Database was accessed and patients without a history of another tumor diagnosed with pathologically confirmed invasive squamous, adenosquamous carcinoma or adenocarcinoma of the cervix between January 2010 and December 2015 who had at least 1 month of follow-up were identified. Patients with pathological stage IB disease (revised 2018 International Federation of Gynecology and Obstetrics staging schema), who underwent radical hysterectomy (as assessed from site-specific surgery codes) with at least 10 lymph nodes removed (all negative based on pathology report, cut-off selected to ensure adequate staging and exclude patients with possible occult lymph node metastases) and had negative tumor margins were selected for further analysis. In the present study, we defined intermediate risk patients as those who had tumor size 2-4 cm with lymph-vascular invasion or tumor size $>4 \mathrm{~cm}$ similar to a prior study and a proposed randomized trial. ${ }^{79}$ Unfortunately, the National Cancer Database does not collect data on the depth of stromal invasion so the traditional Sedlis (Gynecologic Oncology Group 92) criteria could not be applied.

In the National Cancer Database, tumor size is captured primarily from the pathology report and if that is not available, then from imaging or physical examination. Adjuvant radiation therapy was defined as receipt of external beam radiation therapy (with or without vaginal brachytherapy) within 6 months from surgery. Chemotherapy data was captured for patients who received it concurrently during adjuvant radiation therapy. In the present study, exclusion criteria were receipt of neoadjuvant chemotherapy or neoadjuvant radiation therapy, unknown surgery-radiotherapy interval, receipt of radiation therapy $>6$ months from surgery, or receipt of vaginal brachytherapy alone, omission of lymphadenectomy, performance of limited lymphadenectomy $(<10$ lymph nodes removed), positive or unknown tumor margin status, unknown tumor size, and unknown pathological stage. When applying inclusion criteria (except lymphovascular invasion and tumor size), $10.4 \%$ of patients had missing tumor size and $7.8 \%$ had missing lymph-vascular invasion status.

The National Cancer Database is a hospital-based database capturing approximately $70 \%$ of all malignancies diagnosed in the United States. The American College of Surgeons and the Commission on Cancer have not verified and are not responsible for the analytical or statistical methodology employed, or the conclusions drawn from these data. The present study was deemed exempt from Institutional Board Review from Penn Medicine (Protocol \#829268).

Demographic, clinico-pathological, and treatment characteristics were extracted from the de-identified dataset. For analysis purposes, insurance status was recoded into Private, Government (including Medicaid and Medicare), and Uninsured/Unknown. Given the impact of menopause on the overall survival of patients, based on the median age of menopause in the United States, patient age was dichotomized into $<50,>50$ years. The presence of co-morbidities was assessed from the Charlson-Deyo Comorbidity index and classified as absent (score 0 ) or present (score $\geq 1$ ). The type of treatment facility was categorized into academic/research and others that included community cancer program, comprehensive community cancer program, and integrated network cancer program.
The frequency of the distribution of categorical variables was compared with the chi-square test and continuous variables with the Kruskall-Wallis test. Median follow-up in each group was calculated based on the reverse Kaplan-Meier method that is used to assess the length and completeness of the follow-up and is constructed by reversing 'censor' and 'event'. Overall survival was assessed following the generation of Kaplan-Meier curves and compared with the log-rank test. A Cox model was constructed to control for a priori selected confounders (patient age, histology, and surgical approach) known to be associated with overall survival based on prior studies. Stratified analyses by histology, and mode of radical hysterectomy were performed given the possible different biological behavior of cervical cancer histotypes as demonstrated in the ad hoc analysis of the Gynecologic Oncology Group 92 randomized trial, ${ }^{10}$ as well as a recent retrospective study demonstrating possible overall survival benefit of adjuvant radiotherapy only for patients who had minimally invasive hysterectomy. ${ }^{11}$ Statistical analyses were performed with the Statistical Package for the Social Sciences v.27 (International Business Machines Corporation Corporation. Armonk, New York) and the alpha level of statistical significance was set at 0.05 .

\section{RESULTS}

A total of 765 patients who met the inclusion criteria were identified. Median patient age was 43 years (range, 23-90), while the majority of patients were White $(78.2 \%)$, without comorbidities $(86.9 \%)$. The median number of lymph nodes removed and examined was 20 (range, 10-78). The mode of radical hysterectomy was available for 683 patients, in which $50.5 \%$ had a laparotomy. The most common histologic subtype was squamous cell carcinoma (68.5\%), and lymphovascular invasion was present in $65.6 \%$ of patients. In the present cohort, adjuvant external beam radiation therapy was administered to 378 patients (49.4\%). The median interval between surgery and radiation therapy initiation was 54 days (IQR 23). Among patients who received external beam radiation therapy, 102 patients $(27 \%)$ received vaginal brachytherapy while 219 patients (57.9\%) received chemotherapy (189 patients as single agent, 15 patients as multiagent, and 15 patients unknown number of chemotherapy agents). Table 1 summarizes the clinic-pathological characteristics based on the receipt of adjuvant radiation therapy. Patients who received adjuvant radiotherapy were older (median 45 vs 43 years, $P=0.007$ ) and more likely to have lymphovascular invasion $(72.9 \%$ vs $62.9 \%, P=0.004)$. Tumor size, mode of surgery, type of hysterectomy, number of lymph nodes resected, patient race, insurance, and presence of comorbidities were comparable between the two groups.

Median follow-up for patients who did and did not receive adjuvant radiation therapy was 45.01 and 44.75 months, respectively. There was no difference in overall survival between patients who did ( $n=378,37$ deaths) and did not ( $n=387,44$ deaths) receive adjuvant radiation therapy, $\mathrm{P}=0.44$ : 4-year overall survival rates were $88.4 \%$ and $87.1 \%$, respectively (Figure 1). After controlling for patient age, histology, and surgical approach, the administration of adjuvant radiation therapy was not associated with better survival (HR $0.86,95 \% \mathrm{Cl} 0.54$ to 1.38). Following stratification by histology, the administration of adjuvant radiation therapy was not associated 
Table 1 Clinico-pathological characteristics stratified by receipt of adjuvant radiation therapy

\begin{tabular}{|c|c|c|c|}
\hline & $\begin{array}{l}\text { Observation } \\
(n=387)\end{array}$ & $\begin{array}{l}\text { Radiation } \\
\text { therapy }(n=378)\end{array}$ & P-value \\
\hline Age (years) & & & 0.001 \\
\hline$<50$ & $289(74.7 \%)$ & $241(63.8 \%)$ & \\
\hline$>50$ & $98(25.3 \%)$ & $137(36.2 \%)$ & \\
\hline Race & & & 0.27 \\
\hline White & $296(76.5 \%)$ & $302(79.9 \%)$ & \\
\hline Black & $56(14.5 \%)$ & $40(10.4 \%)$ & \\
\hline Other/unknown & 35 (9\%) & $36(9.5 \%)$ & \\
\hline Comorbidities & & & 0.93 \\
\hline No & $336(86.8 \%)$ & $329(87 \%)$ & \\
\hline Yes & $51(13.2 \%)$ & $49(13 \%)$ & \\
\hline Insurance & & & 0.79 \\
\hline Private & $221(57.1 \%)$ & $225(59.5 \%)$ & \\
\hline Government & $133(34.4 \%)$ & $122(32.3 \%)$ & \\
\hline $\begin{array}{l}\text { Uninsured/ } \\
\text { unknown }\end{array}$ & $33(8.5 \%)$ & $31(8.2 \%)$ & \\
\hline Median LNs removed & 20 & 20 & 0.48 \\
\hline Type of hysterectomy & & & 0.39 \\
\hline Modified radical & $56(14.5 \%)$ & $61(16.1 \%)$ & \\
\hline Radical & $229(59.2 \%)$ & $205(54.2 \%)$ & \\
\hline $\begin{array}{l}\text { Radical not } \\
\text { otherwise specified }\end{array}$ & $102(26.4 \%)$ & $112(29.6 \%)$ & \\
\hline Approach & & & 0.32 \\
\hline Open & $186(48.8 \%)$ & $159(52.6 \%)$ & \\
\hline Minimally invasive & $195(51.2 \%)$ & $143(47.4 \%)$ & \\
\hline Histology & & & 0.047 \\
\hline Squamous & $271(70 \%)$ & $253(67 \%)$ & \\
\hline Adenocarcinoma & $97(25.1 \%)$ & $89(23.5 \%)$ & \\
\hline Adenosquamous & $19(4.9 \%)$ & $36(9.5 \%)$ & \\
\hline \multicolumn{2}{|c|}{ Lymph-vascular invasion } & & 0.004 \\
\hline Present & $239(62.9 \%)$ & $263(72.9 \%)$ & \\
\hline Absent & $141(37.1 \%)$ & $98(27.1 \%)$ & \\
\hline Size & & & 0.23 \\
\hline$>2$ to $<4 \mathrm{~cm}$ & 201 (51.9\%) & $180(47.6 \%)$ & \\
\hline$>4 \mathrm{~cm}$ & $186(48.1 \%)$ & $198(52.4 \%)$ & \\
\hline
\end{tabular}

Mode of surgery missing for 82 cases, lymph-vascular invasion 24 cases.

LN, lymph node.

with better survival for patients with squamous $(P=0.17)$, adenosquamous $(P=0.15)$, or adenocarcinoma $(P=0.07)$. Following stratification by mode of surgery, adjuvant radiation therapy was not associated with a survival benefit for patients who had laparotomy $(\mathrm{P}=0.22)$ or a minimally invasive $(\mathrm{P}=0.64)$ approach. Lastly, for patients with lymphovascular invasion, those who received adjuvant radiation therapy $(n=263,21$ deaths) had a trend toward better overall survival compared with those who did not $(n=239$, 33 deaths), $P=0.053$ : 4-year overall survival rates were $90.5 \%$ and $85.8 \%$, respectively (Figure 2).

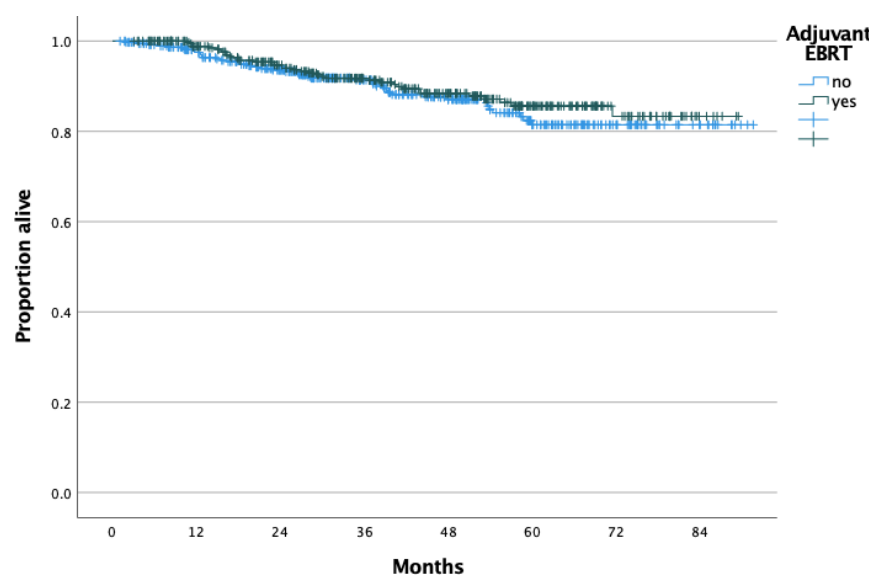

Figure 1 Overall survival of patients with stage IB cervical cancer and intermediate risk factors stratified by receipt of adjuvant external beam radiation therapy.

Among patients who received adjuvant radiation therapy, there was no survival difference between those who did ( $\mathrm{n}=219,19$ deaths) and did not ( $n=159,18$ deaths) receive chemotherapy, $\mathrm{P}=0.36$ : 4-year overall survival rates were $89.8 \%$ and $86.3 \%$, respectively (Figure 3). After controlling for patient age, chemotherapy administration was not associated with better survival (HR $0.75,95 \% \mathrm{Cl} 0.39$ to 1.43$)$.

\section{DISCUSSION}

\section{Summary of Main Results}

In a large cohort of patients with stage IB cervical cancer who underwent radical hysterectomy with negative margins and negative lymph nodes, who had additional negative prognostic factors (such as large size or presence of lymphovascular invasion), half did not receive adjuvant external beam radiation therapy. The administration of adjuvant radiation therapy was not associated with a survival benefit. Similarly, for patients receiving radiation therapy plus chemotherapy, the addition of chemotherapy was not associated with a superior survival. Based on a power calculation, given our current sample size and an overall survival rate of $87 \%$ in the observation alone group, a survival rate of $93 \%$ in the radiotherapy

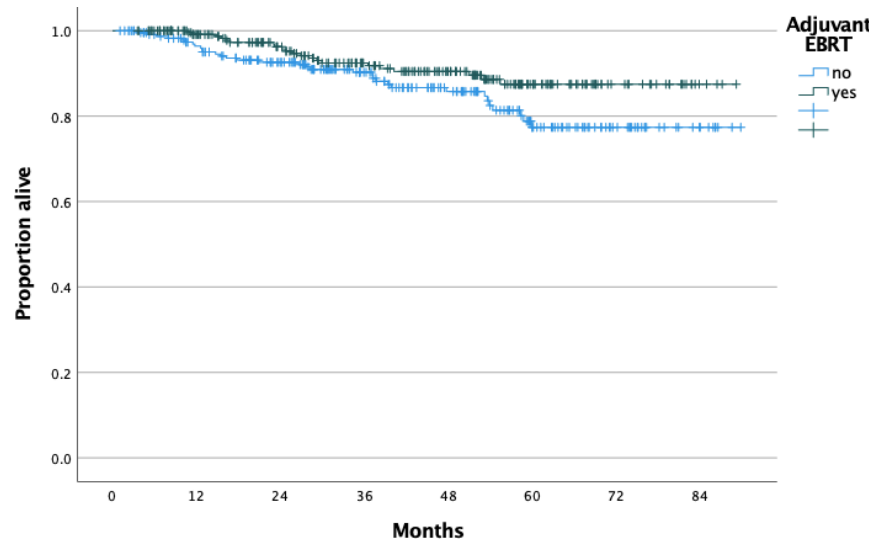

Figure 2 Overall survival of patients with stage IB cervical cancer tumor size $>=2 \mathrm{~cm}$ and lymph-vascular invasion, stratified by receipt of adjuvant external beam radiation therapy. 


\section{Original research}

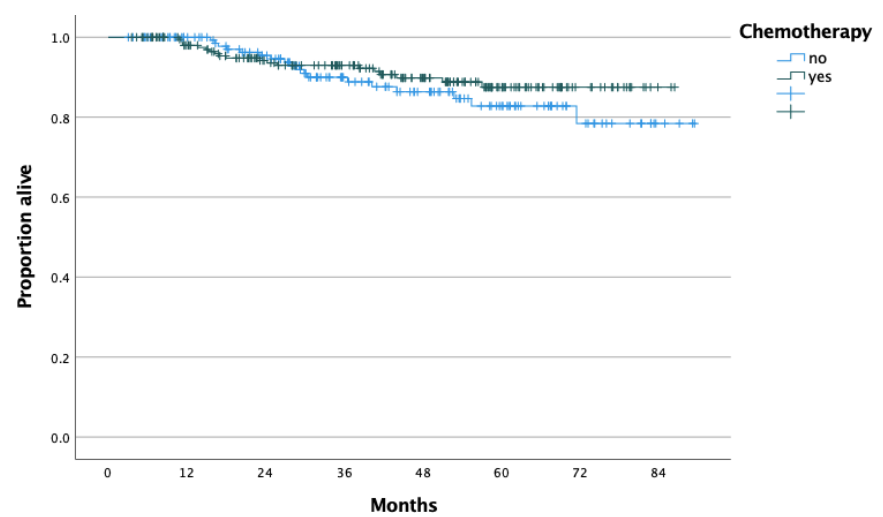

Figure 3 Overall survival of patients with stage IB cervical cancer and intermediate risk factors who received external beam radiation therapy stratified by receipt of chemotherapy.

group would be required to detect a statistical significant difference with an alpha level of 0.05 and an effect size with a HR of 0.52 .

\section{Results in the Context of Published Literature}

The management of patients with lymph node negative, margin negative, early-stage cervical cancer following primary radical hysterectomy has been mostly dictated by the landmark Gynecologic Oncology Group 92 trial. $^{5}$ In that trial, patients who had a combination of additional risk factors, had a significantly lower risk of relapse (15\% vs $28 \%$ ) following adjuvant radiation therapy $(n=137)$ compared with observation alone $(n=140)$. As expected, grade 3 or 4 events were more common in the radiation therapy arm (6\% vs $2.1 \%)$. Interestingly, in a long-term follow-up publication, there was no difference in overall survival between the observation and adjuvant radiation therapy arms (HR: $0.70,95 \% \mathrm{Cl}$ : 0.45 to 1.05$)$. On the other hand, total recurrence rate $(30.7 \%$ vs $17.5 \%, \mathrm{P}=0.007$ ) and progression/death ( $35 \%$ vs $21.9 \%, \mathrm{P}=0.009$ ) rate appeared to favor the radiation therapy arm. ${ }^{11}$ Similarly, progression-free survival was better in the radiation therapy arm (HR: $0.58,90 \% \mathrm{Cl}: 0.40$ to $0.85, \mathrm{P}=0.009$ ). The lack of surgical standardization of a minimum of lymph node count, determination of tumor size by palpation only, and imbalance of risk factors such as lymphovascular invasion or tumor size, between the two groups as well as the relatively high relapse rate in the surgery-only group are the main criticisms of the trial. ${ }^{7}$ Based on the current National Comprehensive Cancer Network guidelines, adjuvant radiation therapy should be considered for patients meeting the Seldis criteria. ${ }^{3}$ Similarly, according to the European Society of Gynaecological Oncology/European Society for Radiotherapy and Oncology/ European Society of Pathology guidelines for the management of patients with cervical cancer, adjuvant radiotherapy should be considered in the presence of a combination of risk factors at final pathology following radical hysterectomy, such as tumor size, lymphovascular invasion, and depth of stromal invasion. ${ }^{4}$ However, based on data from retrospective studies the current European Society of Gynaecological Oncology guidelines state that when an adequate type of radical hysterectomy has been performed observation is an alternative option. ${ }^{412}$

Observation alone has emerged as an acceptable option based on data from retrospective studies from institutions that have not adopted the Sedlis criteria. van der Velden reported the oncologic outcomes of 161 patients from The Netherlands diagnosed between 1982 and 2014 with intermediate-risk cervical cancer based on the Sedlis criteria who did not receive adjuvant radiation therapy following type $\mathrm{C} 2$ radical hysterectomy. ${ }^{8}$ A total of 25 patients $(15.5 \%)$ experienced a relapse while the rate of isolated loco-regional relapse was $5.6 \%$ (nine cases) at a median of 28 months. ${ }^{8}$ Interestingly, the relapse rate was higher for patients with non-squamous histology ( $23.4 \%$ vs $12.3 \%) .^{8}$ Mortality from isolated loco-regional recurrence was low, $2.5 \%$ (four patients). ${ }^{8}$ In another multi-center study, Cibula et al compared the oncologic outcomes of 127 patients with lymph-node negative intermediate risk cervical cancer treated with surgery alone to 104 patients who received chemoradiation. ${ }^{7}$ After a median follow-up of approximately 6 years, rates of relapse ( $6.3 \%$ vs $11.5 \%, P=0.17)$, death (7.1\% vs $14.4 \%, P=0.084)$, and 5 -year overall survival $(94.5 \%$ vs $90.6 \%, P=0.32$ ) were comparable between the two groups. Interestingly, only two isolated pelvic relapses were observed in the surgery-alone group. ${ }^{7}$ Authors attributed the substantially better outcomes observed compared with the Gynecologic Oncology Group 92 trial to more accurate pre-operative and pathological staging as well as improvement in surgical technique. ${ }^{7}$ Ayhan et al also analyzed 393 patients who had lymph node negative stage IB cervical cancer and reported that adjuvant radiation therapy did not provide survival advantage or better local control in that patient population. ${ }^{13}$ In another study from Italy, patients with lymph node negative stage IB-IIA cervical cancer who underwent radical hysterectomy received adjuvant radiation therapy according to personalized indications. ${ }^{14}$ For patients with negative margins who had one or two additional risk factors (lymphovascular invasion and/or $>1 / 3$ of stromal invasion), adjuvant radiation therapy was not associated with better disease-free survival. ${ }^{14}$ In a multicenter retrospective study among 75 patients with stage IB1 disease and intermediate risk factors such as tumor size $2-4 \mathrm{~cm}$, lymphovascular invasion, and/or deep stromal invasion $>1 / 2$, who underwent type III radical hysterectomy between 2005 and 2009 adjuvant treatment was administered to 29 patients (five cases chemoradiation, 19 cases radiation therapy, and five cases chemotherapy alone) while 46 patients did not receive any treatment. ${ }^{15} \mathrm{After}$ an average follow-up of 82.6 months, only one relapse was noted, in a patient who had all three risk factors and had received adjuvant radiation therapy. The incidence of lymphedema was also higher among patients who received chemoradiation or radiation. ${ }^{15}$

The National Comprehensive Cancer Network guidelines suggest that concurrent platinum-containing chemotherapy can be considered for patients with intermediate risk cervical cancer receiving adjuvant pelvic external beam radiation therapy. ${ }^{3}$ This recommendation is extrapolated from data derived from patients with locally advanced stage disease receiving definitive radiation. In our cohort, among patients who received adjuvant external beam radiation therapy, the addition of chemotherapy was not associated with a survival benefit. Similar to our results, a recent retrospective study did not find a survival difference between patients who received radiation alone $(n=243)$ vs chemoradiation $(n=73)$ : after a median follow-up of 70 months 5 -year progression-free $(90.8 \%$ vs $88.9 \%$, $\mathrm{P}=0.63)$ and overall $(95.9 \%$ vs $91 \%, \mathrm{P}=0.29)$ survival rates were comparable between the two groups. ${ }^{16}$ Similarly, a retrospective analysis of a nationwide database from Japan identified 555 patients with intermediate risk stage IB cervical cancer and investigated their outcomes based on the adjuvant treatment strategy: 
$40.2 \%$ received chemotherapy alone, $31 \%$ chemoradiation, and $28.8 \%$ radiation alone. ${ }^{17}$ After controlling for confounders, chemoradiation was not associated with better disease-free (HR: 1.00, $95 \% \mathrm{Cl}: 0.53$ to 1.90 ) or cause-specific (HR: $0.82,95 \% \mathrm{Cl}: 0.34$ to 2.01) survival. ${ }^{17}$ Lastly, in a prior analysis of the National Cancer Database that included 869 patients with stage IB-IIA disease (regardless of tumor size or the presence of lymphovascular invasion) diagnosed between 2004 and 2013, chemoradiation was not associated with better survival compared with radiotherapy alone. ${ }^{18} \mathrm{~A}$ phase 3 randomized trial (NCT01101451) investigating the role of chemoradiation for stage IB-II patients who underwent radical surgery is currently open to enrolment.

In summary, the basis of adjuvant therapy guidelines for intermediate risk early-stage cervical cancer is based on one randomized trial conducted by Sedlis at al, which was published in $1999 .^{5}$ In current times, this has become a continued debate, based on arguments that the current clinical management of cervical cancer has evolved greatly since the Sedlis study was performed three decades ago. These factors include: much more advanced preoperative imaging techniques; pathology-based tumor size measurements; increased ability to identify nodal disease with both imaging and surgical techniques including sentinel lymph node biopsy; and further refinement of surgical radicality definitions. Based on studies by Cibula and van der Velden, which have included more contemporaneously treated patients, these authors have argued and emphasized that the use of of a $\mathrm{C} 2$ radical resection may permit withholding of adjuvant radiation therapy. ${ }^{78}$

In light of our results as well, which captured patients treated in the United States between 2010-2015, we propose that observation may also be considered. However, we do acknowledge there was a worse, though not statistically significant survival difference in those with lymphovascular invasion in our study. Also, as previously mentioned there was a higher recurrence rate in nonsquamous cell $(23 \%)$ vs squamous cell $(12 \%)$ histology in the van der Velden study. These factors should bear caution and require further investigation in future studies of early-stage intermediate risk cervical cancer. Lastly,the extent of surgical radicality required to permit deferring adjuvant radiation must be reviewed. If indeed resection to the pelvic floor as defined by the updated $\mathrm{C} 2$ definition proposed by Querleu et alis required ${ }^{19}$ with no preservation of the autonomic nerves (ie, hypogastric nerve plexus is fully transected), surgeons must be comfortable with this approach and potential functional ramifications (bladder and sexual dysfunction) for the patient.

\section{Strengths and Weaknesses}

A major strength of our study was the large number of patients drawn from a multicenter database. Several limitations of the present study should be mentioned. First, given the lack of a central pathology report, possible tumor and stage misclassifications cannot be excluded. In addition, as previously mentioned, the National Cancer Database does not collect data on the depth of stromal invasion (one of the traditional components of the Sedlis criteria) or data on tumor recurrence and location of relapse. As such, we could not investigate differences in progression-free survival or patterns of relapse. While all patients had negative margins, the radicality of each surgery could not be assessed: in the present study, modified radical hysterectomy corresponded to class B while radical hysterectomy to class $\mathrm{C} 1$ or $\mathrm{C} 2$. Moreover, the presence of unmeasured confounding is a significant limitation of our study. While the vast majority of patients who received chemotherapy were administered a single-agent regimen most likely as a radiosensitizing agent, the exact indications of chemotherapy use are not recorded. Lastly, given its retrospective nature, our study is prone to selection bias: unmeasured factors influencing a clinician's decision to administer adjuvant radiation therapy such as a patient's functional status, specific comorbidities were not available.

\section{Implications for Practice and Future Research}

In a large cohort of patients with lymph node negative, margin negative, intermediate risk stage IB cervical carcinoma, the administration of adjuvant external beam radiation therapy was not associated with a survival benefit compared with observation alone. Future studies evaluating available advanced imaging techniques, the extent of surgical resection in conjunction with observation vs adjuvant therapy, from an international collaborative network are likely required to further elucidate the optimal therapy for this increasingly rare cancer. With great interest we will await the results of the proposed CERVANTES randomized trial. ${ }^{9}$

\section{Conclusions}

In a large cohort of patients with pathological stage IB intermediate risk cervical carcinoma, the administration of adjuvant radiotherapy was not associated with an overall survival benefit.

Contributors DN: conception, data acquisition, data management, statistical analysis, critical analysis, drafting/final editing. NL, AFH, LC, SK, MM, RLGl: critical analysis, drafting/final editing. EMK: supervision, critical analysis, drafting/final editing.

Funding The authors have not declared a specific grant for this research from any funding agency in the public, commercial, or not-for-profit sectors.

Competing interests None declared.

Patient consent for publication Not required.

Provenance and peer review Not commissioned; externally peer reviewed.

Data availability statement Data may be obtained from a third party and are not publicly available. Data are available on request from the American College of Surgeons.

\section{ORCID iD}

Dimitrios Nasioudis http://orcid.org/0000-0001-6260-5353

\section{REFERENCES}

1 Adegoke O, Kulasingam S, Virnig B. Cervical cancer trends in the United States: a 35-year population-based analysis. J Womens Health 2012;21:1031-7.

2 Cervical Cancer Statistics, Centers for Disease Control and Prevention. Available: https://www.cdc.gov/cancer/cervical/ statistics/index.htm [Accessed 10 Dec 2020].

3 Koh W-J, Abu-Rustum NR, Bean S, et al. Cervical cancer, version 3.2019, NCCN clinical practice guidelines in oncology. J Nat/ Compr Canc Netw 2019;17:64-84.

4 Cibula D, Pötter R, Planchamp F, et al. The European Society of Gynaecological Oncology/European Society for Radiotherapy and Oncology/European Society of Pathology guidelines for the management of patients with cervical cancer. Int J Gynecol Cancer 2018;28:641-55.

5 Sedlis A, Bundy BN, Rotman MZ, et al. A randomized trial of pelvic radiation therapy versus no further therapy in selected patients with stage IB carcinoma of the cervix after radical hysterectomy and pelvic lymphadenectomy: a gynecologic Oncology Group study. Gynecol Oncol 1999;73:177-83. 


\section{Original research}

6 Fiorica JV, Roberts WS, Greenberg H, et al. Morbidity and survival patterns in patients after radical hysterectomy and postoperative adjuvant pelvic radiotherapy. Gynecol Oncol 1990;36:343-7.

7 Cibula D, Abu-Rustum NR, Fischerova D, et al. Surgical treatment of "intermediate risk" lymph node negative cervical cancer patients without adjuvant radiotherapy - a retrospective cohort study and review of the literature. Gynecol Oncol 2018;151:438-43.

8 van der Velden J, Mom CH, van Lonkhuijzen L. Analysis of isolated loco-regional recurrence rate in intermediate risk early cervical cancer after a type $\mathrm{C} 2$ radical hysterectomy without adjuvant radiotherapy. Int J Gynecol Cancer 2019;29:874-8.

9 Cervical cancer: adjuvant therapy and surgery (CERVANTES), Gynecologic Cancer Intergroup. Available: https:// gcigtrials.org/system/files/IR\%20CC\%20GCIG\%202019-V5\%20\% 20-\%20\%20Read-Only.pdf [Accessed 10 Dec 2020].

$10 \mathrm{Kim} \mathrm{SI}$, Kim TH, Lee M, et al. Impact of adjuvant radiotherapy on survival outcomes in intermediate-risk, early-stage cervical cancer: analyses regarding surgical approach of radical hysterectomy. J Clin Med 2020;9. doi:10.3390/jcm9113545. [Epub ahead of print: 0311 2020].

11 Rotman M, Sedlis A, Piedmonte MR, et al. A phase III randomized trial of postoperative pelvic irradiation in stage IB cervical carcinoma with poor prognostic features: follow-up of a Gynecologic Oncology Group study. Int J Radiat Oncol Biol Phys 2006;65:169-76.

12 Cibula D. Management of patients with intermediate-risk early stage cervical cancer. J Gynecol Oncol 2020;31:e54.
13 Ayhan A, AI RA, Baykal C, et al. Prognostic factors in FIGO stage IB cervical cancer without lymph node metastasis and the role of adjuvant radiotherapy after radical hysterectomy. Int J Gynecol Cancer 2004;14:286-92.

14 Sartori E, Tisi G, Chiudinelli F, et al. Early stage cervical cancer: adjuvant treatment in negative lymph node cases. Gynecol Oncol 2007;107:S170-4.

15 Nakamura K, Kitahara Y, Satoh T, et al. Analysis of the effect of adjuvant radiotherapy on outcomes and complications after radical hysterectomy in FIGO stage IB1 cervical cancer patients with intermediate risk factors (GOTIC study). World J Surg Oncol 2016;14:173.

16 Kim H, Park W, Kim YS, et al. Chemoradiotherapy is not superior to radiotherapy alone after radical surgery for cervical cancer patients with intermediate-risk factor. J Gynecol Oncol 2020;31:e35.

17 Matsuo K, Shimada M, Yokota $\mathrm{H}$, et al. Effectiveness of adjuvant systemic chemotherapy for intermediate-risk stage IB cervical cancer. Oncotarget 2017;8:106866-75.

18 Mahmoud O, Hathout L, Shaaban SG, et al. Can chemotherapy boost the survival benefit of adjuvant radiotherapy in early stage cervical cancer with intermediate risk factors? a population based study. Gynecol Oncol 2016;143:539-44.

19 Querleu D, Cibula D, Abu-Rustum NR. 2017 update on the QuerleuMorrow classification of radical hysterectomy. Ann Surg Oncol 2017;24:3406-12. 